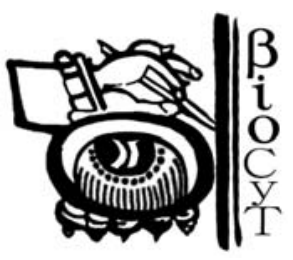

BIOCYT 1(1):1-6, 2008

UNIVERSIDAD NACIONAL AUTONOMA DE MEXICO FACULTAD DE ESTUDIOS SUPERIORES IZTACALA 2010 BIOCYT

http://www.iztacala.unam.mx/biocyt

\title{
EFECTOS DEL RIZOCEFALO Loxothylacus texanus BOSCHMA 1933, SOBRE Callinectes rathbunae CONTRERAS 1930
}

\section{EFFECTS OF RHIZOCEPHALAN Loxothylacus texanus BOSCHMA 1933, ON Callinectes rathbunae CONTRERAS 1930}

\author{
${ }^{1}$ Horacio Vázquez-López y ${ }^{2}$ Fernando Álvarez
}

\begin{abstract}
${ }^{1}$ Laboratorio de Ecología, Facultad de Estudios Superiores Iztacala, Universidad Nacional Autónoma de México. Av. de Los Barrios, No. 1, C.P. 54090, Los Reyes Iztacala, Tlalnepantla, Estado de México, México

${ }^{2}$ Laboratorio de la Colección Nacional de Crustáceos, Instituto de Biología, Universidad Nacional Autónoma de México, Circuito Exterior s/n, Ciudad Universitaria, Copilco, Coyoacán, A. P. 70-153, México, Distrito Federal. C. P. 04510
\end{abstract}

Los crustáceos constituyen un recurso de gran importancia en las pesquerías mundiales; para el caso de nuestro país, la captura de camarón en ambos litorales es una actividad importante; así mismo, dentro de las lagunas costeras la captura de langostinos, jaibas y otros cangrejos representa una actividad significativa para los pescadores locales.

En las lagunas costeras del golfo de México sin embargo, uno de los factores bióticos mas importantes que afectan a las poblaciones de jaibas Callinectes sapidus Rathbun, 1896 (jaiba azul), es el parasitismo causado por rizocéfalos (Crustacea: Rhizocephala), debido a su aparición periódica, alta frecuencia y efectos sobre sus hospederos (Andersen et al., 1990; Alvarez y Calderón, 1996), donde se han atribuido importantes pérdidas económicas a L. texanus (Wardle y Tirpak, 1991; Hochberg et al., 1992).

Los rizocéfalos comprenden cerca de 250 especies de las cuales todas son parásitas, singularmente especializadas de otros crustáceos. Sus hospederos son decápodos o excepcionalmente isópodos, cumáceos, estomatópodos o percebes balanomorfos. Al parecer, los rizocéfalos se encuentran en todos los ambientes acuáticos que habitan sus hospederos, incluyendo el mar profundo (Lutzen, 1985). Algunas especies parasitan cangrejos semiterrestres y otros se encuentran en cangrejos dulceacuícolas (Andersen et al., 1990; Høeg, 1992), por razones aun desconocidas, estos organismos no afectan a las langostas.

Correspondencia al autor: Laboratorio de Ecología, Facultad de Estudios Superiores Iztacala, Universidad Nacional Autónoma de México. Av. de Los Barrios, No. 1, C.P. 54090, Los Reyes Iztacala, Tlalnepantla, Estado de México, México. Correo electrónico: hvazquez@campus.iztacala.unam.mx

Manuscrito recibido el 10 de enero de 2008, aceptado el 05 de marzo de 2008 
El ciclo de vida de los rizocéfalos esta altamente modificado comparado con los percebes de vida libre. No obstante, los rizocéfalos exhiben las dos formas larvales dioicas típicas de los percebes: el nauplio (Fig. 1) y la cipris (Fig. 2) (Høeg, 1991). En pocos géneros, una interna da origen a varias externas. La externa contiene los órganos reproductores y las cavidades del manto (estructura utilizada como incubadora) (Figs. 3 y 4), las cuales en la mayoría de las especies se abren al exterior por medio de un orificio simple en el manto. Un tallo angosto une a la externa con el sistema interno de raíces denominado interna.

Los rizocéfalos al llegar al estado adulto difieren de otros cirripedios por carecer de segmentación, apéndices y tracto digestivo. Los adultos también carecen de cubierta calcárea presente en la mayoría de los cirripedios torácicos.

El rizocéfalo L. texanus, parásito de la jaiba azul (C. sapidus), aparece principalmente en estuarios norteños del golfo de México (Wardle y Tirpak, 1991). Los rizocéfalos parasitan a especies del mismo género de cangrejos (Høeg, 1995).

Desde hace mucho tiempo, los pescadores locales han reconocido la presencia de $L$. texanus en los cuerpos de agua costeros del golfo de México y solo recientemente se han comenzado a estudiar las poblaciones de jaibas afectadas por estos (Lázaro-Chávez et al., 1996; Alvarez y Calderón, 1996; Alvarez et al., 1999).

En México, la pesca de la jaiba es de suma importancia, debido a su amplia aceptación y gran demanda en el mercado nacional, que genera fuentes de trabajo para los pescadores (Rocha-Ramírez et al., 1992). En México, se tiene el registro de once especies del género Callinectes; entre estas, C. sapidus, C. rathbunae Contreras 1930 (jaiba prieta) y C. similis Williams 1966 (jaiba enana) predominan en el golfo de México (Williams, 1974, 1984); en éste, C. sapidus y C. rathbunae soportan una importante pesquería(Alvarez y Calderón, 1996; Alvarez et al., 1999; Vázquez-López et al., 2006; Vázquez-López y Alvarez, 2007) , sin embargo C. rathbunae es la jaiba menos estudiada.

C. rathbunae es una especie (Raz-Guzmán et al., 1992)que se distribuye del sur de Texas, Estados Unidos a la laguna de Términos en el estado de Campeche, México (Williams, 1974; Powers, 1977; Raz-Guzmán et al., 1992; Rocha et al., 1992; Cházaro-Olvera et al., 2000). La mayor abundancia de C. rathbunae ha sido registrada en la laguna de Alvarado, estado de Veracruz, México (Chávez y Fernández, 1976; Rosas, 1989).

En años recientes la pesquería de jaibas se ha incrementado en México debido principalmente a la imposición de la temporada de veda al camarón (Cházaro-Olvera, 2002).

Los efectos que causa este cirripedio parásito a sus hospederos se pueden citar como castración parasítica, cese de muda, hiperfeminización, cambio conductual, ceguera y parálisis (Vázquez-López et al., 2006; Vázquez-López datos no publicados).

La castración parasítica es un proceso mediante el cual el parásito provoca una atrofia a los gonopodios del hospedero mientras que el abdomen se ensancha asemejándose al abdomen de una hembra madura sana (Figs. 5, 6 y 7), por lo cual pierde la posibilidad de reproducirse., L. texanus al igual que la mayoría de las especies de rizocéfalos, tienen la capacidad de afectar el proceso de muda con lo que se detiene el crecimiento de los cangrejos afectados, las hembras parasitadas sufren una hiperfeminización, refiriéndose con esto a que el abdomen sufre un ensanchamiento exagerado, aunado a esto, los pleopodos se ven atrofiados. En este tipo de organismos las gónadas también se ven afectadas lo que conlleva a que la tasa de fecundidad de los hospederos se reduzca a cero (Reinhard, 1956; O’Brien y Van Wyk, 1984; Høeg, 1995; Alvarez y Calderón, 1996; Alvarez et al., 1999; Vázquez-López et al., 2006; Vázquez-López datos no publicados). 
Estos parásitos modifican el comportamiento de los cangrejos braquiuros machos afectados, se ha observado que los organismos con externas, presentan el mismo comportamiento de las hembras ovígeras. Estos acicalan constantemente a la externa como si se tratara de una masa ovígera al mismo tiempo que mueven el abdomen para circular agua y de esta manera suministrar oxígeno a los supuestos huevos (Vázquez-López et al., 2006; Vázquez-López datos no publicados).

Recientemente Vázquez-López et al., (2006) y Vázquez-López (datos no publicados) han observado que jaibas de la especie $C$. rathbunae parasitadas con $L$. texanus que presentan tres y cuatro externas maduras, sufren una especie de ceguera, puesto que en todos los experimentos realizados, al momento de suministrarles alimento, estos organismos presentaron movimientos erráticos al dirigirse al mismo. Tales movimientos incluyeron contactos fuertes y constantes contra las paredes de los acuarios en los que se encontraban alojadas. Se observó que los mismos organismos no presentaron movimientos de los apéndices natatorios, los cuales siempre se alojaron sobre el caparazón.

Un efecto a nivel poblacional de este parasitismo es que la fracción parasitada de jaibas, la cual no se reproduce, compite por espacio y alimento con los individuos no parasitados (Alvarez y Calderón, 1996).

Un efecto para las pesquerías locales es que los individuos parasitados no alcanzan la talla legal de captura. Los pescadores de jaibas argumentan que los organismos capturados parasitados por rizocéfalos (conocidos localmente por los pescadores como jaibas de carterita o de bolsita) son descartados ya que resultan mas difíciles de procesar para la obtención de pulpa (carne de jaiba vendida a granel) porque los caparazones resultan mas resistentes.

Por lo anterior, es importante realizar en México estudios integrales sobre la biología del cangrejo $C$. rathbunae. Sobre los efectos que causa $L$. texanus en su hospedero, existe poca información, pudiéndose citar los trabajos de Alvarez y Calderón (1996), Alvarez et al. (1999), Vázquez-López et al. (2006) y Vázquez-López y Alvarez (2007).

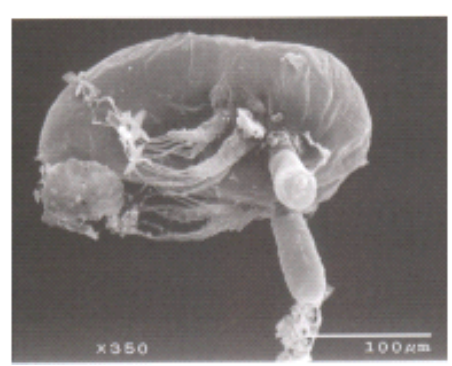

Fig. 1. Larva nauplio de L. Texanus

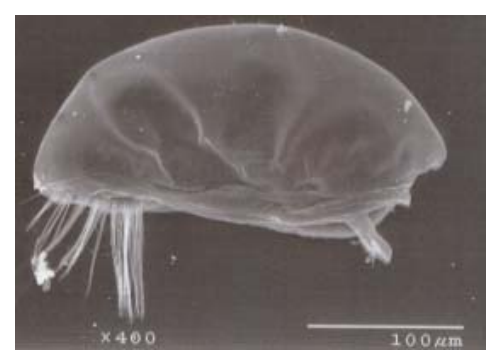

Fig. 2. Larva cipris de L. texanus 


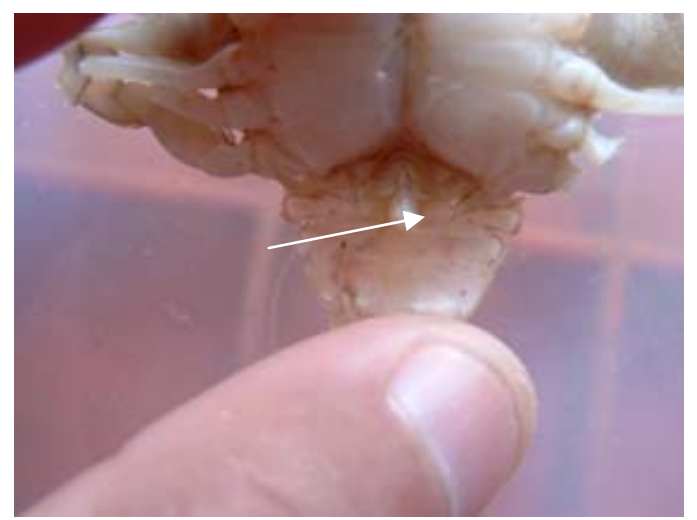

Fig. 3. Externa virgen del rizocéfalo L. texanus en su hospedero C. rathbunae (indicado con flecha).

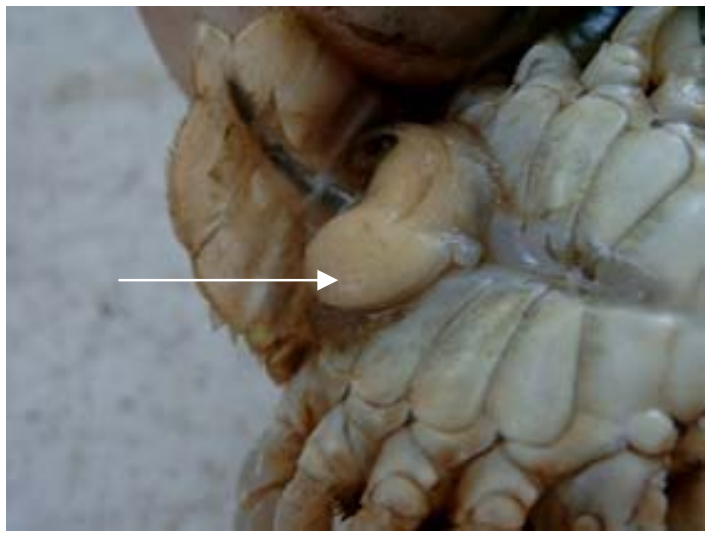

Fig. 4. Externa madura del rizocéfalo L. texanus (indicado con flecha).

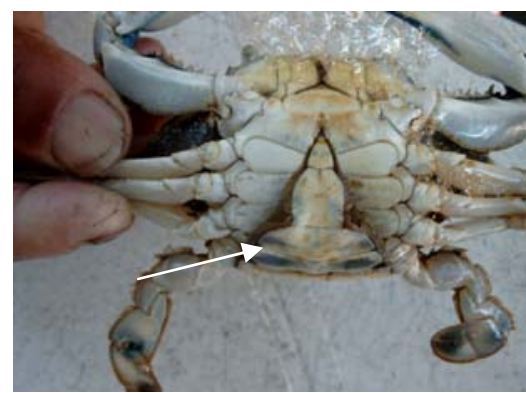

Fig. 5.

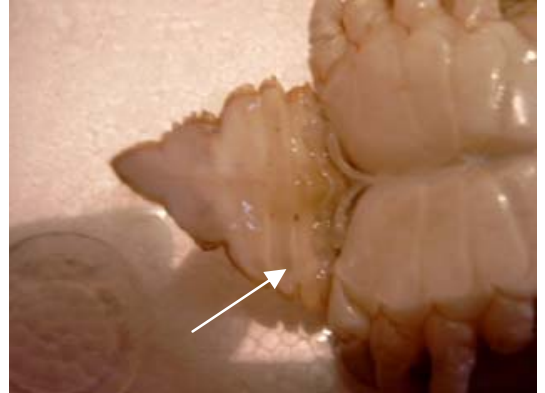

Fig. 6.

Fig. 5. Macho feminizado (en la parte media del abdomen se observa un ensanchamiento) (señalado con flecha).

Fig. 6. El abdomen de un macho parasitado es semejante al abdomen de una hembra inmadura sana (señalado con flecha). 

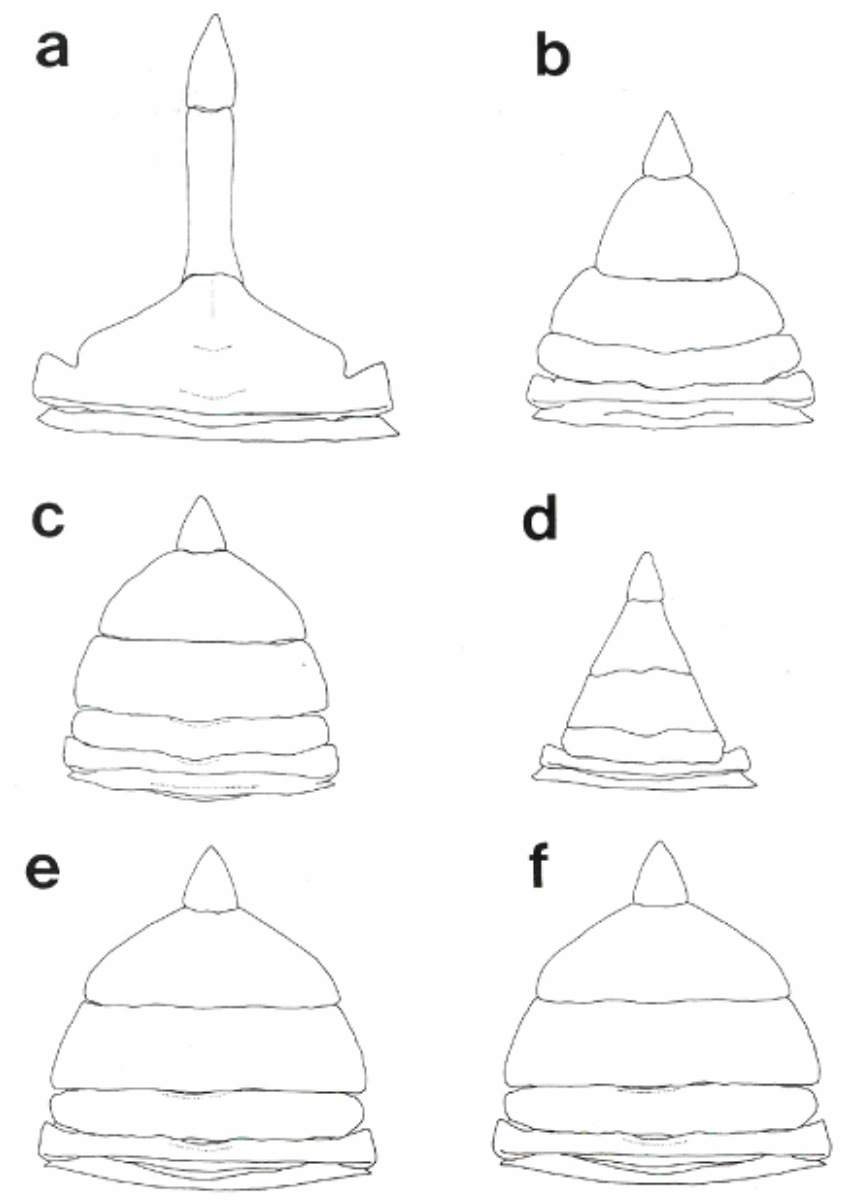

Fig. 7. Abdomen de C. rathbunae: a) macho normal, b) macho parasitado con abdomen triangular, c) macho parasitado con abdomen redondeado, d) hembra inmadura, e) hembra madura normal, y f) hembra parasitada. Tomado y modificado de Alvarez y Calderón, 1996.

\section{REFERENCIAS}

Alvarez, F. y J. Calderón, 1996. Distribution of Loxothylacus texanus (Cirripedia: Rhizocephala) parasitizing crabs of the genus Callinectes in the southwestern Gulf of México. Gulf Research Reports, 9: 205-210.

Alvarez, F., A. Gracia, R. Robles y J. Calderón, 1999. Parasitization of Callinectes rathbunae and Callinectes sapidus by the rhizocephalan barnacle Loxothylacus texanus in Alvarado Lagoon, Veracruz, México. Gulf Research Reports, 11: 15-21.

Andersen, M. L., M. Bohn, J. T. Høeg y P. G. Jensen, 1990. Cyprid ultrastructure and adult morphology in Ptychascus barnwelli new species and P. glaber (Cirripedia: Rhizocephala), parasites on semiterrestrial crabs. Journal of Crustacean Biology, 10: 20-28.

Chávez, E. y M. S. Fernández, 1976. Contribución al conocimiento de la biología de la jaiba prieta (Callinectes rathbunae: Decapada, Portunidae), del estado de Veracruz. Revista de la Sociedad Mexicana de Historia Natural, 27: 237-291.

Cházaro, O. S., R. A. Rocha y L. H. Vázquez, 1999. Cultivo de jaiba: Una nueva perspectiva en acuacultura. Revista de Zoología, 7: 40-43. 
Cházaro, O. S., A. Rocha-Ramírez y R. Román-Contreras, 2000. Observations on feeding, maturity and fecundity of Callinectes similis Williams, 1966, on the central continental shelf off Veracruz, Gulf of Mexico. Crustaceana, 73: 323-332.

Cházaro-Olvera, S., 2002. Efecto de la salinidad sobre la tasa de crecimiento de Callinectes sapidus Rathbun y Callinectes rathbunae Contreras. 151 p. (Tesis doctoral, Facultad de Ciencias, Universidad Nacional Autónoma de México, México).

Hochberg, R. J., T. M. Bert, P. Steele y S. D. Brown, 1992. Parasitization of Loxothylacus texanus on Callinectes sapidus; aspects of population biology and effects on host morphology. Bulleti of Marine Science, 50: 117-132.

Høeg, J. T., 1992. Microscopic anatomy of invertebrates. En: Harrison, F. W. y Humes, A. G. (Eds).Crustacea. Wiley-Liss, New York. Pp 313-345.

Høeg, J. T., 1995. The Biology and life cycle of the Rhizocephala (Cirripedia). Journal of the Marine Biological Association of the United Kingdom, 75: 517-550.

Lázaro-Chávez, E., F. Alvarez y C. Rosas, 1996. Records of Loxothylacus texanus_(Cirripedia: Rhizocephala) parasitizing the blue crab Callinectes sapidus in Tamiahua Lagoon, México. Journal of Crustacean Biology, 16: 105-110.

O’Brien, J. y Van Wyk, P., 1984. Effects of crustacean parasitic castrators (epicaridean isopods and rhizocephalan barnacles) on growth of their crustacean hosts. En: Wenner A. M. (Ed.). Crustacean issues, 3. Factors in adult growth. A. A. Balkema Press, Rotterdam, The Netherlands. Pp 191-218.

Powers, L. H., 1977. A catalogue and bibliography to the crabs (Brachyura) of the Gulf of México. Contributions in Marine Science, (suppl.), 20: 1-190.

Raz-Guzmán, X., J. Sánchez y A. Soto, 1992. Catálogo ilustrado de cangrejos brachyuros y anomuros (Crustácea) de la laguna de Alvarado, Veracruz, México. Serie Cuadernos, 14. Instituto de Biología, Universidad Nacional Autónoma de México. 51 p.

Reinhard, E. G., 1956. Parasitic castration of Crustacea. Explorations on Parasitology, 5: 79-107.

Rocha-Ramírez, A., S. Cházaro-Olvera y P. M. Mueller-Meier, 1992. Ecología del género Callinectes (Brachyura: Portunidae) en seis cuerpos de agua costeros del Estado de Veracruz, México. Anales del Instituto de Ciencias del Mar y Limnología, 19. Universidad Nacional Autónoma de México. Pp 33-41.

Rosas, V. C., 1989. Aspectos de la ecofisiología de las jaibas Callinectes sapidus, Callinectes rathbunae y Callinectes similis de la zona sur de la laguna de Tamiahua, Veracruz (Crustacea; Decapada; Portunidae). 200 p. (Tesis doctoral, Facultad de Ciencias, Universidad Nacional Autónoma de México, México).

Vázquez-López, H., F. Alvarez, J. Franco, A. Morán y S. Cházaro, 2006. Observations on the behavior of the dark crab Callinectes rathbunae Contreras parasitized with the rhizocephalan Loxothylacus texanus Boschma. International Journal of Zoological Research, 2(4): 344-353.

Vázquez-López, H. y F. Alvarez, 2007. Space-temporal presence of the cirripede parasite Loxothylacus texanus in the Lagoon-Estuarine Subsystem of Alvarado, Veracruz, México. International Journal of Zoological Research, 3(4): 157-168.

Wardle, W. J. y A. J. Tirpak, 1991. Occurrence and distribution of an outbreak infection of Loxothylacus texanus (Rhizocephala) in blue crabs of Galveston Bay, Texas, with special reference to size and coloration of the parasite's external reproductive structures. Journal of Crustacean Biology, 11: 553-560.

Williams, A. B., 1974. The swimming crabs of the genus Callinectes (Decapoda: Portunidae). Fishery Bulletin, United States, 70(2/3): 685-369.

Williams, A. B., 1984. Shrimp, lobsters and crabs of the Atlantic coast of the eastern United States, Maine to Florida. Smithsonian Institution Press, Washington, D. C. 550 p.

BIOCYT (Biología, Ciencia y Tecnología), año 3, número 11, julio-septiembre 2010. Publicación trimestral editada en la Facultad de Estudios Superiores Iztacala, UNAM. Avenida De Los Barrios, número 1, Los Reyes Iztacala, Tlalnepantla, Estado de México, México, C. P. 54090. Teléfono: 5623 11 73. Dirección electrónica: http://www.iztacala.unam.mx/biocyt

Editor responsable: Horacio Vázquez López. Reservas de Derechos al Uso Exclusivo número: 04-2009-121617011000-203, ISSN: en trámite con número de folio 018439, ambos otorgados por la Comisión Calificadora de Publicaciones y Revistas Ilustradas de la Secretaría de Gobernación. Responsable de la última actualización de este número biólogo José Ángel Lara Vázquez, Cabecera de la Carrera de Biología de la FES Iztacala, Edificio de Gobierno. Fecha de última actualización, 03 de agosto de 2010. Las opiniones expresadas por los autores no reflejan necesariamente la postura del editor de la revista.

Se autoriza la reproducción (sin fines de lucro) total o parcial de los contenidos e imágenes de la revista, citando la fuente completa y la dirección electrónica 